\title{
Fatal pulmonary embolism and coincidental cerebral infarction after spinal anesthesia
}

\section{-A case report-}

\author{
Jong-Yeon Lee, Su-Yeon Lee, Inho Shin, Chunghyun Park, Byung-Sang Lee, and Min Sung Kim \\ Department of Anesthesiology and Pain Medicine, School of Medicine, CHA University, Pocheon, Korea
}

\begin{abstract}
A pulmonary embolism and cerebral infarction are the second and third most common acute cardiovascular diseases after a myocardial infarction. Early diagnosis and appropriate management are important clinical challenges. In this case, a fatal pulmonary embolism and extensive cerebral infarction caused cardiac arrest during spinal anesthesia for total hip replacement surgery. Transesophageal echocardiography indicated a pulmonary embolism and brain CT showed large area of acute infarction at right middle cerebral artery territory. Pulmonary CT angiogram revealed massive pulmonary embolism findings. This paper reviews this case and suggests other preventive modalities. (Korean
\end{abstract} J Anesthesiol 2011; 61: 515-518)

Key Words: Cerebral infarction, Pulmonary embolism, Spinal anesthesia.

Pulmonary embolism (PE) and cerebral infarction (CI) are the second and third most common acute cardiovascular diseases after myocardial infarction [1]. PE is one of the most important causes of postoperative morbidity and mortality [2]. Early diagnosis and appropriate management are important clinical challenges. A range of preoperative evaluations and prevention are believed to decrease the morbidity and mortality. Over the last 10 years, pulmonary CT angiogram (PCTA) has been highlighted as a diagnostic tool of PE rather than conventional pulmonary angiogram or ventilation perfusion scan. It is less invasive, gives an immediate result, and is highly specific and sensitive method. This paper describes a case of coincidental PE and extensive CI causing cardiac arrest during spinal anesthesia, and suggests other preventive modalities.

\section{Case Report}

An 80-year-old woman, $155 \mathrm{~cm}$ in height and $70 \mathrm{~kg}$ in weight, was admitted to our institution via the emergency room due to a right femur fracture. The patient had a 5-year history of hypertension, which was relatively well controlled with the medication regimen of an antihypertensive agent and aspirin. She did not exercise much due to obesity and depression. She showed delirium symptoms after admission and underwent brain computerized tomography (CT) to exclude organic brain

Received: March 2, 2011. Revised: April 2, 2011. Accepted: April 26, 2011.

Corresponding author: Min Sung Kim, M.D., Department of Anesthesiology and Pain Medicine, School of Medicine, CHA University, 351, Yatap-dong, Bundang-gu, Seongnam 463-712, Korea. Tel: 82-31-780-5557, Fax: 82-31-701-9433, E-mail: eldorado100@naver.com (c) This is an open-access article distributed under the terms of the Creative Commons Attribution Non-Commercial License (http:// creativecommons.org/licenses/by-nc/3.0/), which permits unrestricted non-commercial use, distribution, and reproduction in any medium, provided the original work is properly cited. 
diseases. A brain CT showed severe stenosis of the middle cerebral artery (MCA), an old localized infarction of the basal ganglia, and diffuse atrophy of the brain. She exhibited cardiomegaly on the preoperative chest X-ray, 1 degree A-V block on EKG, an ejection fraction of $65 \%$ on echocardiography and other blood studies, and her vital signs are within the normal limits. The preoperative PT/PTT was 12.7/24.4 seconds, INR was 1.15 , and arterial blood gas analysis (ABGA) revealed the following: pH 7.376, $\mathrm{PaO}_{2} 78.6 \mathrm{mmHg}, \mathrm{PaCO}_{2} 27.5 \mathrm{mmHg}$.

On the $4^{\text {th }}$ hospital day, she was scheduled to undergo total hip replacement surgery and aspirin was maintained until the $3^{\text {rd }}$ hospital days. Upon arrival at the operating theater, standard monitoring devices were applied and the left radial artery was cannulated for continuous blood pressure monitoring. The patient's blood pressure (BP), heart rate (HR) and $\mathrm{O} 2$ saturation was $140 / 70 \mathrm{mmHg}, 80$ beats/min, and $98 \%$, respectively, through a simple facial mask with $4 \mathrm{~L} / \mathrm{min}$ of oxygen. Spinal anesthesia was administered with the patient in the right lateral position using a 26-gauge Quincke spinal needle at the L3-4 interspace with $0.5 \%$ hyperbaric bupivacaine $8 \mathrm{mg}$ under standard aseptic conditions. The patient was then placed in the supine position. During the pin-prick test, the patient became unresponsive with BP 70/40 mmHg and HR 40-45 beats/min. Repeated ephedrine administration intravenously (IV) followed by epinephrine 10 $\mu \mathrm{g}$ and full dripping of the crystalloid did not increase the BP. Endotracheal intubation was performed after $7-8$ minutes of manual ventilation and norepinephrine $0.1 \mu \mathrm{g} / \mathrm{kg} / \mathrm{min}$, dopamine $2 \mu \mathrm{g} / \mathrm{kg} / \mathrm{min}$ were administered continuously The patient's BP and HR was still 60/40 mmHg and 110 beats/min, respectively, and IV epinephrine $0.02 \mu \mathrm{g} / \mathrm{kg} / \mathrm{min}$ was started.

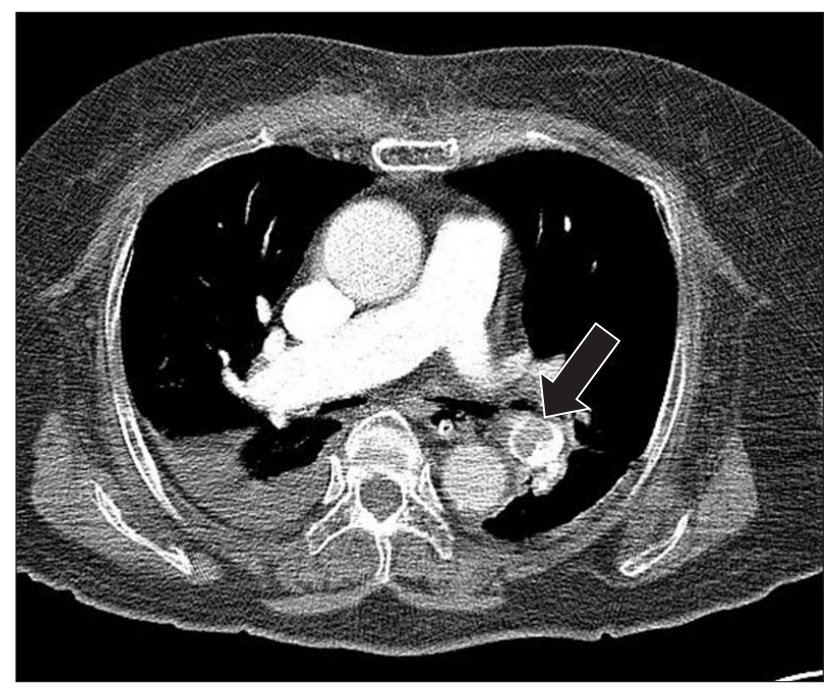

Fig. 1. Enhanced chest CT scan at the level of right main pulmonary artery shows low density ovoid thromboembolism from lobar pulmonary artery of left lower lobe to entire segmental pulmonary artery of left lower lobe.
The ABGA at that time revealed the following: $\mathrm{PaO}_{2}=488.9$ $\mathrm{mmHg}, \mathrm{PaCO}_{2}=30.8 \mathrm{mmHg}$ at $\mathrm{FiO}_{2}$ 1.0, and $\mathrm{EtCO}_{2}=28 \mathrm{mmHg}$ and $\mathrm{O} 2$ saturation $=99 \%$. The cause was suspected to be high spinal anesthesia rather than a pulmonary embolism because of the ABGA findings. Central venous catheterization via the right internal jugular vein was performed to administer the drugs and for hemodynamic monitoring. The central venous pressure was $30 \mathrm{mmHg}$ and emergent TEE was performed immediately. TEE revealed an enlargement of the right atrium (RA) and right ventricle (RV), straightening of the interventricular septum and hypokinesia, which were consistent with PE. IV heparine 5,000 $\mathrm{IU}$ was administered and maintained at 1,000 IU/hr. $50 \mu \mathrm{g} / \mathrm{kg}$ of milrinone IV was loaded for 10 minutes and maintained at 0.5 $\mu \mathrm{g} / \mathrm{kg} / \mathrm{min}$ to manage RV failure. The ABGA results exhibited pH 7.224, $\mathrm{PaO}_{2} 55.2 \mathrm{mmHg}$ and $\mathrm{PaCO}_{2} 52.8 \mathrm{mmHg}$, which were also consistent with PE.

The operation was postponed and the patient was transported to the intensive care unit (ICU). Twenty minutes later, her heart beat decreased to 30 beats/min and cardiopulmonary resuscitation was performed. Chest 3 dimensional PTCA and non-contrast brain CT were performed when the patient's hemodynamic parameter became temporarily stable.

A low density ovoid thromboembolism from the lobar pulmonary artery of the left lower lobe to the entire segmental pulmonary artery of the left lower lobe and enlargement of the right atrium and ventricle, which suggested a poor prognosis, was observed on the enhanced PCTA (Fig. 1). In addition, a low density large acute infarct at the right MCA territory with gyral swelling of the right insula and temporal lobe were noted on the non-enhanced brain CT scan (Fig. 2). After 17 hours admission to the ICU, her heart rate decreased to 30 beats $/ \mathrm{min}$,

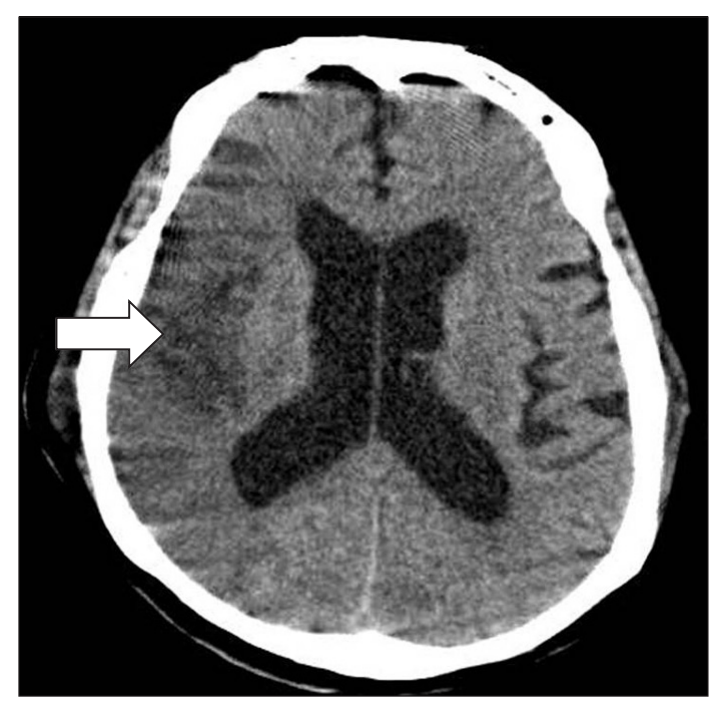

Fig. 2. Non-enhanced brain CT scan shows low density acute infarct with gyral swelling of right insula and temporal lobe. 
resuscitation was ineffective and she died.

\section{Discussion}

PE would be a fatal disease unless a prompt treatment should be made. In particular, it is known to commonly occur following the onset of fracture of lower extremities and the surgery. Other risk factors include an age of 40 years or older, a long-term bed rest, malignant tumor, multiple trauma, a past history of PE, obesity, congestive heart failure and a long-term use of estrogen [3]. PE and CI, occurring during anesthesia or surgery, always make anesthesiologists or surgeons bewildered. Besides, they are also fatal diseases that raise the mortality or morbidity.

In the current case, not a single presence of PE, it is assumed that PE occurred immediately after or almost concurrently to the onset of CI. Cases in which the blod clots formed from the deep vein thrombosis (DVT) might cause a concurrent presence of cerebral infarction and PE correspond to those in which there is a patent foramen ovale (PFO) [4]. In the current case, no PFO was preoperatively detected on echocardiography. It might therefore be plausible to consider that the current case originates from two different etiologic factors. In addition, if there is a single presence of $\mathrm{PE}$, the arterial $\mathrm{CO}_{2}$ concentration would be increased and the end-tidal $\mathrm{CO}_{2}$ would be decreased prior to the occurrence of a loss of the mentality. This would also be accompanied by hypoxia due to respiratory difficulty. In the current case, however, a loss of the mentality firstly occurred. Immediately after the endotracheal intubation, both $\mathrm{PaO}_{2}$ and $\mathrm{PaCO}_{2}$ were within a normal range. Based on these findings, it is presumed that acute $\mathrm{CI}$ was a pre-existing condition. As the causative factors that are responsible for the occurrence of CI in the current case, there are such ones as the fluctuation of blood pressure or changes in the hemodynamic status. As the atherosclerotic plaque was broke down, the cerebral infarction occurred due to the MCA stenosis which was preoperatively observed on brain CT scans. It may be probable that CI due to the decreased blood pressure occurs in patients with cerebral artery stenosis as shown in the current case. Nevertheless, it is noteworthy that CI mainly occurs in the end artery. On brain CT scans which were performed at an ICU, however, there were findings that are suggestive of a diffuse presence of acute CI in the right MCA territory. These findings lead to the speculation that the acute CI could not occur due to the fluctuation of blood pressure. Because CI occurring during spinal anesthesia is an unpredictable condition, however, we could not completely ruled out the possibility that a high-spinal anesthesia was involved.

The increased central venous pressure is a typical finding that is suggestive of pulmonary infarction due to the PE. The first reason that we suspected $\mathrm{PE}$ as an etiologic factor in the current case is a marked increase of the central venous pressure by approximately $30 \mathrm{mmHg}$. A trans-esophageal echocardiography (TEE), which is commonly performed at an operation room, is of help in making the early treatment with a prompt identification of PE. The dilatation of right atrium and right ventricle, the formation of linearity in the ventricular septum due to a strong shift to the left side and the embolism formed in the right ventricle correspond to typical findings. In cases of a smaller embolism, however, no typical findings occur. In cases in which the embolism occurred in the pulmonary vessels, no typical findings might be observed in the right ventricle [5]. In the current case, no embolism was seen in the right ventricle. Because there were typical findings of $\mathrm{PE}$, however, the treatments for PE could be initiated immediately. In cases of PE, both fat embolism and thrombus embolism can occur. In the current case, however, as shown in Fig. 1, there was a diffuse presence of PE in the left main pulmonary artery, the right lobar artery and both descending arteries. These findings lead to the speculation that the current case arises from the PE due to the thrombus.

Cerebral infarction that occurred in the current case had a severe degree. In elderly patients, however, there is a brain atrophy. It can therefore be inferred that the brain edema does not cause a death because it compresses the brain stem. Accordingly in the current case, the death cause was a typical case of PE. This type of embolism might occur in patients aged 40 years or older, those who had a fracture of the lower extremities ad those who were at secondary risk factors such as a more than 3-day bed rest or bedridden or immobilization [6]. In addition, there is also a possibility that PE progresses over several days and then this leads to the occurrence of a fulminant case of PE. In these cases, if a diagnosis of PE should be preoperatively made using computed tomography pulmonary angiography, this would be useful in preventing the occurrence of fatal case of PE leading to the death with the use of more active treatment regimens such as the preoperative use of anti-coagulation therapy or thrombolysis, embolectomy and inferior vena cava (IVC) filter. This is quite regrettable. It has been reported that the thrombus was degraded because of the vasodiation due to anesthesia and the fluid infusion for anesthesia in patients who had spinal anethesia following the onset of the fracture of femur [7]. It is generally known that local anesthesia produced DVT or PE at a lower incidence as compared with general anesthesia. But this is because the formation of new blood clots is decreased and local anesthesia has a great effect on the pre-existing presence of blood clots [8].

Due to the increased number of elderly people in whom there is a concurrent presence of cardiovascular disease, cerebrovascular disease and coronary artery disease, intraoperative 
PE or perioperative, acute CI have been reported to frequently occur. It is also expected that they would further increasingly occur [9]. In the field of internal medicine or emergeny medicine, in the early 2000s, the PTCA has been used to make a diagnosis of PE at the same ratio to ventilation-perfusion lung scan. But a ventilation-perfusion lung scan shows the specificity in cases in which the pulmonary perfusion was decreased for any reasons. In patients with pulmonary disease who concurrently had chronic inflammation, however, the pulmonary hemodynamics would be decreased and this would greatly lower the specificity even if the blood vessels around the lung should undergo atrophy. In addition, an emergency test would also be unavailable. From a cost-effective perspective, it is not a preferrable to the PCTA. Since 2006, PCTA has become a mainstream modality that is used in most of the patients who are suspected to have PE. The sensitivity and specificity of PCTA have been reported to reach $90 \%$ and $95 \%$, respectively [10]. Due to an excessive use of PCTA in patients who are suspected to have PE, however, there were such problems as cost-ineffectiveness and the toxicity of contrast medium in patients with renal dysfunction. Accordingly, the indications of PCTA have been standardized based on such methods as Wells scoring system or Geneva scoring system (GSS) [2]. According to the GSS, patients aged between 60 and 79 years were given 1 point, those aged 80 years or older were given 2 points, those who previously had PE or DVT were given 2 points, those who had a past history of taking surgery were given 3 points, those whose heartbeat exceeded 100 times/min were given 1 point, those whose $\mathrm{PaCO}_{2}$ was lower than $36 \mathrm{mmHg}$ were given 2 points, those whose $\mathrm{PaCO}_{2}$ ranged between 36 and $39 \mathrm{mmHg}$ were given 1 point, those whose $\mathrm{PaO}_{2}$ was lower than $49 \mathrm{mmHg}$ were given 4 points, those whose $\mathrm{PaO}_{2}$ ranged between 49 and $60 \mathrm{mmHg}$ were given 3 points, those whose $\mathrm{PaO}_{2}$ ranged between 61 and $71 \mathrm{mmHg}$ were given 2 points and those whose mmHg $\mathrm{PaO}_{2}$ ranged between 71 and $82 \mathrm{mmHg}$ were given 1 point. Besides, patients who findings that are suggestive of platelike atelectasis or elevation of hemidiaphragm on a chest $\mathrm{X}$-ray were given 1 point each. To summarize, patients whose scores ranged between 0 and 4 points, those whose scores ranged between 5 and 8 points and those scores exceeded 9 points were classified into a low probability, a moderate probability and a high probability [1]. The current case was given 5 points based on the age and the arterial blood gas analysis, which corresponds to a moderate probability. In all the cases in which a moderate probability was determined based on the GSS, it would be unacceptable to perform a PCTA for a preventive use. In the current case, however, a relevant test would be of further help. This is also quite regrettable.

Diagnostic modalities or antibiotic treatments, which are preoperatively performed to identify and then to treat the thrombus, do not guarantee a $100 \%$ success rate of prevention. It is presumed, however, that the preoperative use of PCTA might be of help for the prevention of severe PE to some extents for patients who are at increased risks of developing thrombus formation. In addition, a meticulous monitoring of clinical course, a prompt diagnosis or an accurate treatment at an operation room would be mandatory.

\section{References}

1. Stein PD, Matta F. Acute pulmonary embolism. Curr Probl Cardiol 2010; 35: 314-76.

2. Goldhaber SZ. Pulmonary embolism. Lancet 2004; 363: 1295-305.

3. Torbicki A, Perrier A, Konstantinides S, Agnelli G, Galie N, Pruszczyk $\mathrm{P}$, et al. Guidelines on the diagnosis and management of acute pulmonary embolism: the Task Force for the Diagnosis and Management of Acute Pulmonary Embolism of the European Society of Cardiology (ESC). Eur Heart J 2008; 29: 2276-315.

4. Islam MA, Khalighi K, Goldstein JE, Raso J. Paradoxical embolismreport of a case involving four organ systems. J Emerg Med 2000; 19: 31-4.

5. Wilson WC, Frankville DD, Maxwell W, Carpenter T, Hastings RH. Massive intraoperative pulmonary embolus diagnosed by transesophageal echocardiography. Anesthesiology 1994; 81: 504-8.

6. Dehring DJ, Arens JF. Pulmonary thromboembolism: disease recognition and patient management. Anesthesiology 1990; 73: $146-64$.

7. Lee JJ, Shin BS, Hong JS. Pulmonary thromboembolism following spinal anesthesia. Korean J Anesthesiol 1999; 36: 534-9.

8. Bullingham A, Strunin L. Prevention of postoperative venous thromboembolism. Br J Anaesth 1995; 75: 622-30.

9. Kyrle PA, Eichinger S. New diagnostic strategies for pulmonary embolism. Lancet 2008; 371: 1312-5.

10. van Belle A, Buller HR, Huisman MV, Huisman PM, Kaasjager $\mathrm{K}$, Kamphuisen PW, et al. Effectiveness of managing suspected pulmonary embolism using an algorithm combining clinical probability, D-dimer testing, and computed tomography. JAMA 2006; 295: 172-9. 